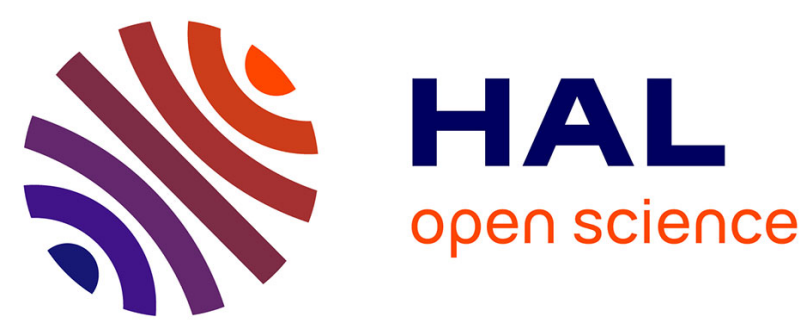

\title{
Integrating markov chain models and L-systems to simulate the architectural development of apple trees
}

\author{
M. Renton, Evelyne Costes, Christophe Godin, Yann Guédon
}

\section{To cite this version:}

M. Renton, Evelyne Costes, Christophe Godin, Yann Guédon. Integrating markov chain models and L-systems to simulate the architectural development of apple trees. 7th International Symposium on Computer Modelling in Fruit Research and Orchard,, 2006, France. pp.63-70. hal-00120798

\section{HAL Id: hal-00120798 https://hal.science/hal-00120798}

Submitted on 18 Dec 2006

HAL is a multi-disciplinary open access archive for the deposit and dissemination of scientific research documents, whether they are published or not. The documents may come from teaching and research institutions in France or abroad, or from public or private research centers.
L'archive ouverte pluridisciplinaire HAL, est destinée au dépôt et à la diffusion de documents scientifiques de niveau recherche, publiés ou non, émanant des établissements d'enseignement et de recherche français ou étrangers, des laboratoires publics ou privés. 


\title{
Integrating Markov Chain Models and L-systems to Simulate the Architectural Development of Apple Trees
}

\author{
M. Renton*, E. Costes*, Y. Guédon** and C. Godin** \\ *UMR BEPC INRA/AgroM/CIRAD/IRD, \\ Equipe Architecture et Fonctionnement des Espèces Fruitières, Montpellier, France \\ ** UMR AMAP CIRAD/INRA/IRD/CNRS/UMII, \\ Botanique et Bioinformatique de l'Architecture des Plantes, Montpellier, France
}

Keywords : branching, growth, fruit trees, 3D simulation, hidden semi-Markov chain

\begin{abstract}
An approach for modelling and simulating the architectural development of apple trees is presented. The approach is based on using an L-systems framework to integrate a Markov model of terminal bud fate and a number of hidden semiMarkov models of axial bud fate. Results show that these models are able to represent the branching zones observed in apple trees at node scale, simulate the sequences of annual growth units found along branches, and capture important aspects of the bearing pattern. Integrating these models gives a simulation of the development of the whole tree that can be used to evaluate the ability of local-scale models to capture global characteristics.
\end{abstract}

\section{INTRODUCTION}

When studying fruit trees, it is important to consider the architecture or structure of the tree, in addition to more commonly studied issues such as fruit quality and resistance to pest and disease (Laurens et al., 2000). In the context of orchard management, the structure of the tree will have a strong influence on the amount of tree maintenance required, with certain tree forms reducing the need for pruning and chemical thinning. The branching pattern of the tree is also closely related to the important issue of the regularity of fruit bearing (Lauri et al., 1997).

Previous work on apple trees (Malus $x$ domestica Borkh) has investigated intraspecific variability in apple tree architecture (Costes and Guédon 1997, 2002, Costes et al., 2003). These studies were based on a method for measuring and analysing plant architecture that combines topological and geometrical descriptions of plants at several scales simultaneously (Godin et al., 1997, 1999). Local-scale empirical models were developed for analysing branching patterns along the trunks of different apple cultivars (Costes and Guédon 1997, 2002). A succession of distinct homogenous zones along the trunk was observed and represented in a relatively empirical way by Markov models (Guédon et al., 2001). These Markov models were also applied to analysing the zones observed in shoots of different lengths in peach trees (Fournier et al., 1998).

On the other hand, L-systems provide a well-established approach for modelling the development of branching systems (Prusinkiewicz and Lindenmayer, 1990). They have been used in agronomic and horticultural applications such as simulating the structural development of cotton plants and peach trees, based on functional hypotheses (Hanan and Hearn, 2002 , Allen et al., 2004). 
The goal of the present study is to further develop the approach used for the trunk by parameterising Markov chain models for branching along the other annual growth units (GUs) of the tree. We also aim to extend the approach to model the succession of GUs along branches. These local-scale models will then be integrated into a global simulation of the architectural development of whole apple trees, based on L-systems.

The initial aim of the study was to produce a relatively accurate whole tree simulation so that comparison between global characteristics of the simulated trees and the measured trees could be used to evaluate the ability of local-scale Markovian models to capture global characteristics of the trees. We therefore used a stochastic approach rather than a detailed modelling of the underlying functional-structural mechanisms, such as carbon allocation or hormone interaction. A long term goal is to produce an accurate simulation, which could be used to predict the architectural development of different cultivars over time, and thus investigate and specify inter-cultivar differences in fruit position and regularity. We were interested in whether an approach based on Markov chain models and L-systems would be capable of producing such a simulation.

\section{MATERIALS AND METHODS}

The general approach, as illustrated in Figure 1, is to start by analysing a database of branching structures and thus formulating local-scale stochastic models of branching based on Markov chains. Using L-systems, these local-scale models will then be integrated into a simulation of full tree architectural development. Simulated structures can then be compared to the original structures at the global scale.

Constructing the simulation involved finding ways of answering a number of questions. When is a bud transformed into a growing apex? How many internodes and axial buds will a growing apex produce before becoming a dormant terminal bud? What will be the fate of this terminal bud? What will be the fate of the axial buds? The answers to these questions are based on the system of categorisation used in the tree structure database, and the local-scale Markovian models built from this database.

\section{Encoded Database of Measured Tree Structures}

The database consists of recorded measurements for two six-year-old Fuji apple trees, with development over the six years deduced by using morphological markers such as leaf scars (Costes et al., 2003). From this data base, GUs of different types were coded using numerical symbols. Proleptic vegetative short, medium and long branches were represented with a 1, 5 and 2, respectively, and sylleptic GUs with a 4. Each bud was assigned a code according to its fate, so that a bud that produced a long GU was a type 2 bud, for example. Dormant buds were represented with a 0 .

This categorisation defined the timing for growth within the simulation. Buds of type 1,2 and 5 grow in the following year, unless they are the 'terminal' bud of a floral GU (the bourse shoot bud), in which case they grow immediately (Crabbé and Escobedo, 1991). Buds of type 4 (sylleptic) always grow immediately.

This categorisation also enabled the extraction of sequences representing the fate of the axillary buds along a GU, or the succession of GUs (fate of terminal buds) along a growth axis. These sequences could then be analysed to produce local-scale Markovian models of terminal and axillary bud fate. 


\section{Local-Scale Markovian Models of Bud Fate}

1. Markovian Model for Terminal Bud Fate. A stochastic Markovian model was used to predict the fate of terminal buds and thus the sequence of GU types along an axis. The basic model formulation is illustrated graphically in Figure 2. Formally, a Markov chain consists of a set of states, a set of probabilities governing which will be the initial or starting state, and a matrix of probabilities controlling the transitions between states. (Ephraim and Merhav, 2002). In this case, the 'states' correspond to the possible fates of a terminal bud: a long GU, a short GU, a medium GU, a floral GU or death. The type of the first GU of an axis is given by the hidden semi-Markov models described below, except for the trunk. The fate of the terminal bud is then controlled by a matrix of transition probabilities obtained by an analysis of the observed sequences of GUs along the axes. For example, the transition probability from a long GU to a floral GU in the model is given by calculating the proportion of long GUs in the database for which the following GU was floral. (GUs not followed by another GU were not considered). Based on previous data analysis (Costes et al., 2003), the probability of death each year is 0.3 for short GUs and zero for other GUs.

2. Hidden Semi-Markov Models for Axillary Bud Fate. A preliminary analysis of the observed sequences representing the fates of the axillary buds along a GU suggested a succession of distinct homogenous zones along all the GUs in the tree, similar to that observed for the trunk. For example, we might find a zone of mostly floral GU buds, followed by a zone of mostly dormant buds, followed by a zone of mixed long and medium GU buds. This in turn suggested the use of hidden semi-Markov chain (HSMC) models to predict these sequences. Formally, a hidden semi-Markov chain consists of a number of states, a set of initial probabilities and a matrix of transition probabilities, like a Markov chain. In addition, each state has an occupancy distribution and an observation distribution associated with it. In this case, the 'states' correspond to distinct homogenous zones along a GU; the initial and transition probabilities control the succession of zones found along a GU; the occupancy distribution controls the length of the zones; and the observation distribution controls the fates of the axillary buds within the zone, as shown in Figure 3. In the simulation, a previously developed HSMC model was adapted for use for the trunk (Costes and Guédon, 2002), and new models were developed for the branches.

\section{L-systems}

Formally, an L-system consists of an alphabet of symbols (which can represent parts of a plant), an axiom (or starting point) and productions or re-writing rules. Figure 4 gives a simple example of how symbols and re-writing rules, combined with a graphical translation, can be used to represent plant development. (Lindenmayer and Prusinkiewicz, 1990).

The basic symbols used in the apple tree simulation are $I$ (internode), $A$ (growing apex) and $B$ (dormant bud). Dormant buds $B$ may be transformed into growing apices $A$, which produce a number of internodes $I$ and axillary buds $B$, before transforming again into a dormant terminal bud $B$. The fate of the terminal bud is determined by the Markov model. The type of all the axillary buds produced by the apex is determined by the relevant HSMC model when the apex is created. Buds of type 4 (sylleptic) are transformed into a growing apex immediately. Buds of type 1,2 and 5 produce a growing apex in the following year, unless they are the 'terminal' bud of a floral GU (the bourse 
shoot bud), in which case they are transformed immediately. Buds of type 0 remain dormant.

Visualisation of the L-system simulation requires the specification of geometrical characteristics such as branching and phyllotactic angles. Initially these angles were chosen to give a purely schematic and 2-dimensional visualisation of tree topology. In order to produce more visually realistic tree forms, a series of simple geometrical hypotheses was then introduced. Under these hypotheses, the initial form of the tree is based on branching and phyllotactic angles chosen based on observation of real tree forms and a tropism that causes branches to grow upwards. This form is then dynamically modified by the hypotheses that branches that are more than one year old will bend down, representing the effect of the weight of more distal growth and fruits.

\section{RESULTS}

\section{Parameterisation of Terminal Bud Fate Model}

Table 1 gives the transition probabilities for the Markovian model of terminal bud fate resulting from analysing the sequences of GU types along growth axes. Notice that all vegetative GUs are most likely to be followed by a floral GU, with the highest observed probability being for the medium GUs. About two thirds of floral GUs are followed by a short GU and one third by a medium GU. Long GUs are almost always found only after other long GUs, reflecting the fact that long GUs tend to occur mostly in the first years of growth.

\section{Number of Nodes in GUs}

The lengths of the sequences representing bud fate along a GU varied widely, as shown in Figure 5, which made it impractical to model all sequences with a single HSMC model. We therefore decided to divide the GU sequences into categories according to their length. It was assumed that sequences less than 5 nodes long corresponded to short GUs, those between 5 and 15 nodes long corresponded to medium GUs and those greater than 16 nodes long corresponded to long GUs. The long GU sequences were then further divided into three categories according to node number (16-25, 26-40, 41+). Separate HSMC models were then built to simulate the sequences from each category.

Analysis showed that the proportions of GUs in these categories varied widely according to the year of growth, as shown in Figure 6. Therefore, in the simulation, the probability that a given new long GU will belong to a certain length category varies with the growth year, according to these probabilities.

\section{Hidden Semi-Markov Models}

The hidden semi-Markov modelling of the branching along GUs revealed the presence of distinct zones. General patterns could be observed in the hidden semi-Markov models. The model for the initial trunk was relatively complex and contained seven states, whereas for shorter GUs simpler models with fewer states were adequate. For example, Figure 7 shows the simplest model for the category of 15-25 node long GUs. This model accounts for the possibility of having floral and non-floral GUs.

We also tried categorizing GUs by year and constructing distinct HSMC models for each year. The small number of 26-40 and $41+$ GUs for some years made this yearly categorising impractical for these categories. However, the HSMC models for the medium and 16 to 25 categories revealed a yearly effect regarding the floral zone and the 
probability of flowering within the zone, reflecting the alternative bearing behaviour of Fuji.

\section{Simulation}

The integration of the parameterised local-scale models using the L-systems formalism results in a dynamic, visual, node-by-node representation of the architectural development of the apple trees. Figure 8 shows an example of how the tree structure is built up annually by the addition of new GUs. Figure 9 shows how the stochastic nature of the simulation results in a range of different structures, corresponding to the range of structures found in the orchard for the same genotype under the same conditions, and also the result of applying the simple geometrical hypotheses discussed previously to obtain more realistic tree forms.

\section{DISCUSSION}

This paper presents the intermediate results of a work in progress. We have shown that integrating local-scale Markov chain models with L-systems provides a method for simulating the dynamic topological development of Fuji apple trees. The addition of simple geometrical hypotheses gives a reasonable representation of the way that the trees' form develops over time. However, the effect of reducing the number and complexity of the underlying HSMC branching models should be investigated. This will indicate whether overall model complexity can be reduced without compromising accuracy at the global level.

The next step of model validation will involve evaluating the effectiveness of the local-scale stochastic modelling and the general approach of integrating these local-scale models into a global simulation. To address this issue, we intend to compare simulated tree structures with measured tree structures at a whole-tree-scale, looking at global characteristics such as the total number of different GU types over several years, with a focus on the regularity of floral GUs. In order to explore this regularity in greater depth, we will also consider more complex Markov models with memory greater than one.

We will then investigate the sensitivity of the global model output to changes in parameters of the local-scale models. For example, these investigations will show how important the terminal bud fate model is in terms of simulated global development. This could lead to greater understanding of the importance of terminal bud fate in the development of real trees.

When the general approach has been satisfactorily validated and refined for Fuji, it will then be applied to simulating the development of Braeburn apple trees. This will show whether the approach is applicable to different varieties and whether it is able to simulate important intra-specific differences. This would indicate that the approach could be a valuable tool for quantifying and analysing the structural development and bearing patterns of fruit trees.

\section{Literature Cited}

Allen, M., Prusinkiewicz, P. and DeJong, T. 2004. Using L-Systems for modeling the architecture and physiology of growing trees: The L-PEACH model. In: C. Godin et al. (eds.) Proc. of the 4th Intern. Workshop on Functional-Structural Plant Models. Montpellier, France. 
Costes, E. and Guédon, Y. 1997. Modeling the sylleptic branching on one-year-old trunks of apple cultivars. Journal of the American Society of Horticultural Science 122: 5362.

Costes, E. and Guédon, Y. 2002. Modelling branching patterns in 1-year-old trunks of six apple cultivars. Annals of Botany 89 (5): 513-524.

Costes, E., Sinoquet, H., Kelner, J.J. and Godin. C. 2003. Exploring within-tree architectural development of two apple tree cultivars over 6 years. Annals of Botany 91 (1): 91-104.

Crabbé, J. and Escobedo-Alvarez. J. A. 1991. Activités méristématiques et cadre temporel assurant la transformation florale des bourgeons chez le Pommier (Malus $x$ Domestica Borkh., cv. Golden Delicious). L'Arbre: Biologie et developpement. Naturalia Monspeliensia: 369-79.

Ephraim, Y. and Merhav, N. 2002. Hidden Markov processes. IEEE Transactions on Information Theory 48, 1518-1569.

Fournier, D., Guédon, Y. and Costes, E. 1998. A comparison of different fruiting shoots of peach trees. IVth ISHS Peach Symposium. Bordeaux (France). Acta Horticulturae 465: 557-65.

Godin, C., Guédon, Y., Caraglio, Y. and Costes, E. 1997. Measuring and analysing plants with the AMAPmod software. In : Plants to Ecosystems. Australia, CSIRO: 53-84.

Godin, C., Costes, E. and Sinoquet, H. 1999. A method for describing plant architecture which integrates topology and geometry. Annals of Botany 84 (3): 343-357.

Guédon, Y., Barthélémy, D., Caraglio, Y. and Costes, E. 2001. Pattern analysis in branching and axillary sequences. Journal of Theoretical Biology 212 (4): 481-520.

Hanan, J. and Hearn. A.B. 2002. Linking physiological and architectural models of cotton. Agricultural Systems 14: 1-31.

Jirasek, C., Prusinkiewicz, P. and Moulia, B. 2000. Integrating biomechanics into developmental models expressed using L-systems. In: H.-Ch. Spatz and T. Speck (eds.): Plant Biomechanics 2000. Proceedings of the 3rd Plant Biomechanics Conference, Freiburg-Badenweiler, August 27 to September 2, 2000. Georg Thieme Verlag, Stuttgart: 615-624.

Laurens, F., Audergon, J.M., Claverie, J., Duval, H., Germain, E., Kervella, J., Lelezec, M., Lauri, P.E. and Lespinasse., J.M. 2000. Integration of architectural types in French programmes of ligneous fruit species genetic improvement. Fruits 55: 141-52.

Lauri, P.E., Terouanne, E. and Lespinasse, J.M. 1997. Relationship between the early development of apple fruiting branches and the regularity of bearing - An approach to the strategies of various cultivars. Journal of Horticultural Science 72: 519-30.

Prusinkiewicz, P. and Lindenmayer, A. 1990. The Algorithmic Beauty of Plants. New York, Springer.

\section{Table}

Table 1. Terminal Bud Fate Probabilities.

\begin{tabular}{|l|c|c|c|c|c|}
\cline { 3 - 7 } \multicolumn{2}{c|}{} & \multicolumn{5}{|c|}{ Following GU } \\
\cline { 3 - 7 } \multicolumn{2}{c|}{} & Short & Long & Floral & Medium \\
\hline \multirow{3}{*}{$\begin{array}{l}\text { Preceding } \\
\text { GU }\end{array}$} & Short & 0.30 & 0.01 & 0.64 & 0.05 \\
\cline { 2 - 7 } & Long & 0.02 & 0.28 & 0.56 & 0.14 \\
\cline { 2 - 7 } & Floral & 0.67 & 0.05 & 0 & 0.27 \\
\cline { 2 - 7 } & Medium & 0.07 & 0.04 & 0.78 & 0.11 \\
\hline
\end{tabular}




\section{$\underline{\text { Figures }}$}

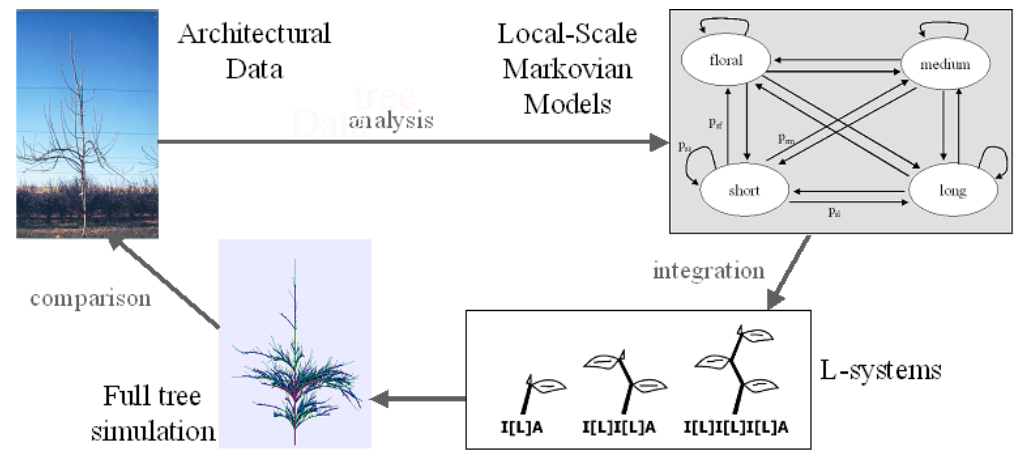

Fig. 1. Schematic representation of the general approach.

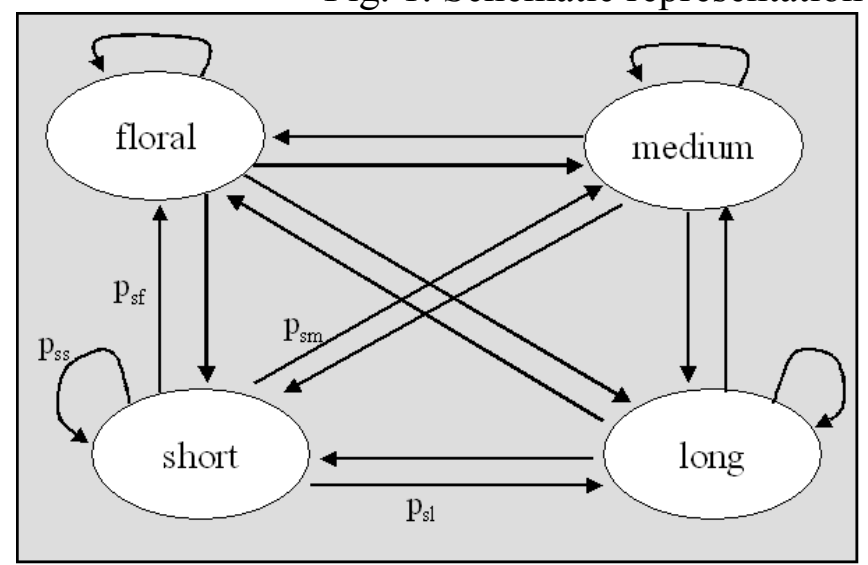

Fig. 2. Markovian model of terminal bud fate. The ovals correspond to the possible growth units. Model parameterisation involves assigning a transition probability to each arrow. For example, $\mathrm{p}_{S f}$ is the probability that the terminal bud of a short GU will produce a floral GU. The sum of probabilities for a given GU type (e.g. $p_{s f}, p_{s s}, p_{s m}$, and $\left.p_{s l} \mathrm{f}\right)$ must equal one. Some of these transition probabilities may actually be close to or equal to zero.

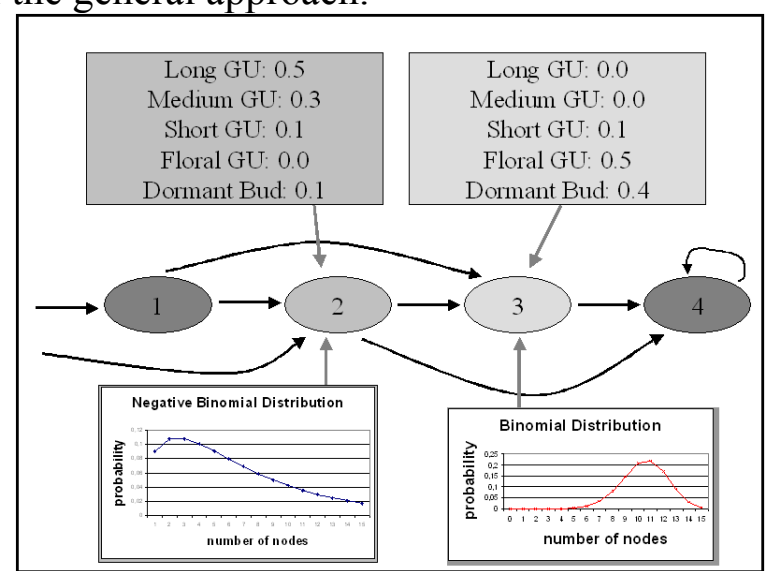

Fig. 3. An example of a hidden semi-Markov model for axillary bud fate along a GU. The states (ovals) correspond to homogenous zones of nodes along a GU. Black arrows indicate the transition from one zone to another and has an associated probability. Each zone has an associated observation distribution (boxes above) and occupancy distribution (plot), illustrated here for zones 2 and 3 .

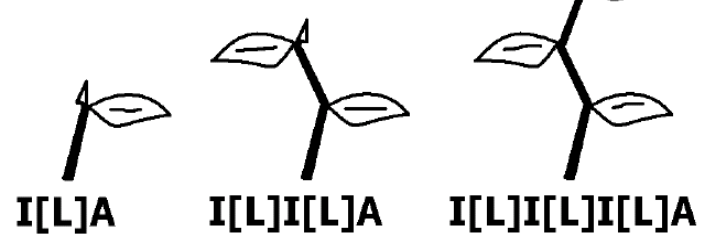

Fig. 4. Using L-systems to represent plant components and development with symbols. In this case the axiom is $I[L] A$, where $I$ represents an internode, $L$ stands for a leaf, $A$ is an apical meristem. The square brackets enclose branching structures. At each step the $A$ is rewritten as $I[L] A$ and in the corresponding graphical translation the small triangle representing an apical meristem is replaced with a new internode, a leaf and a new apical meristem. 


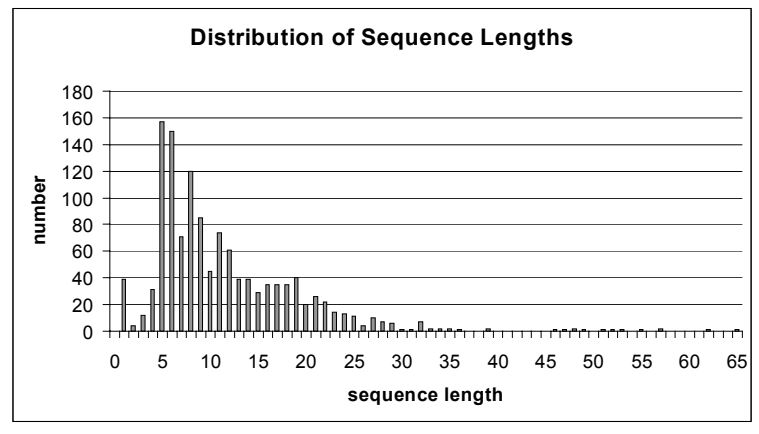

Fig. 5. The distribution of the lengths of the sequences representing the fate of the buds along the different GUs.

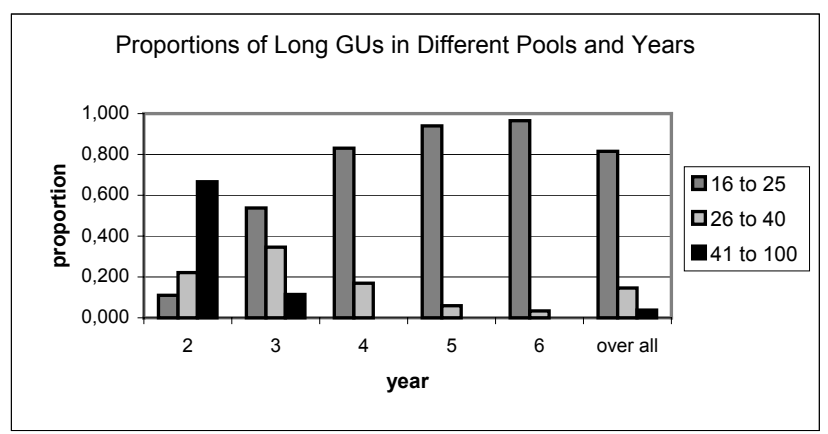

Fig. 6. Proportions of long GUs in different length categories according to growth year.

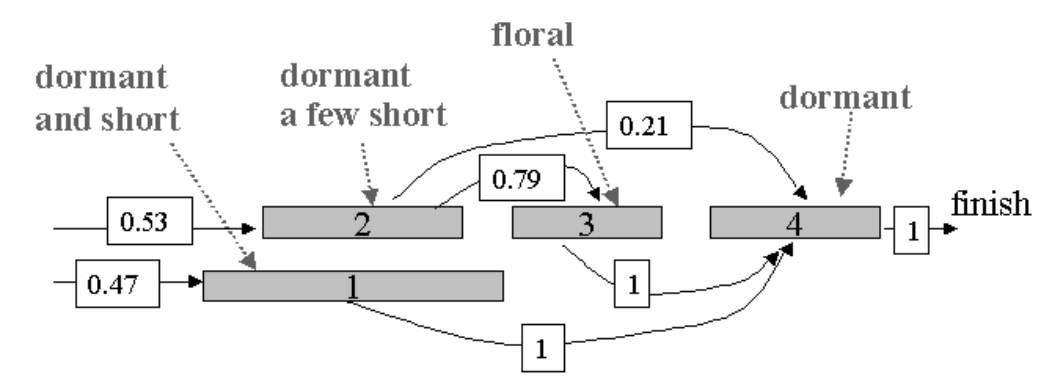

Fig. 7. Graphical representation of a parameterised four state hidden semi-Markov model for long GUs between 15 and 26 nodes long. The grey boxes represent the states of the model, corresponding to four possible branching zones. The length of the box corresponds to the average length of the zone and the dominant type of bud is indicated for each zone. Transition probabilities are also shown.
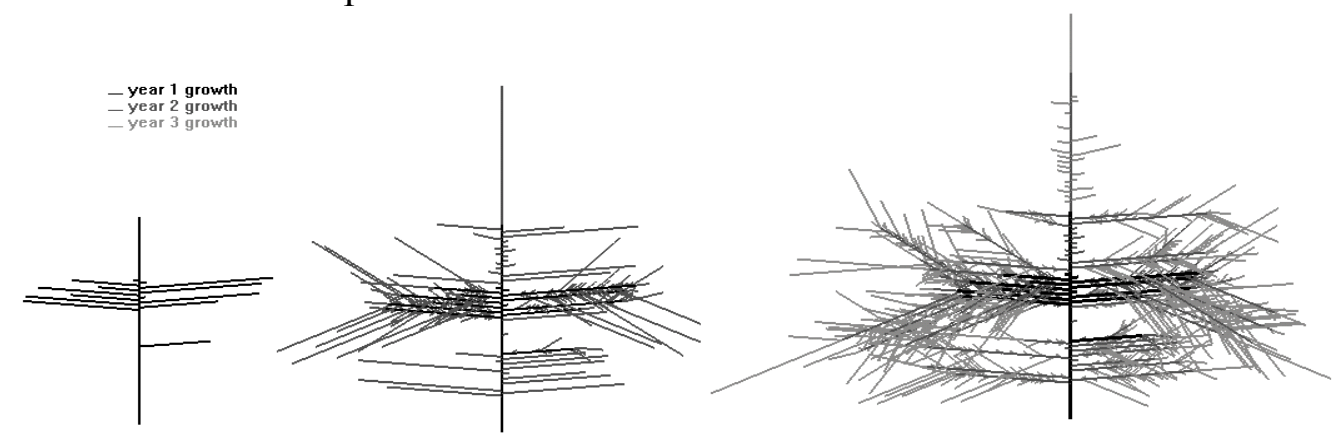

Fig. 8. Schematic representation of simulated tree structure at the end of one, two and three years.
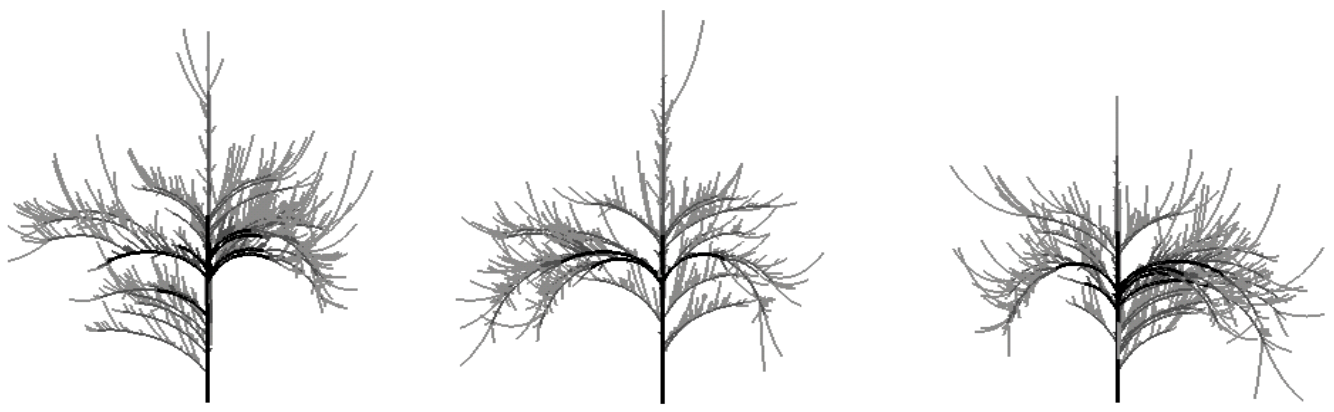

Fig. 9. Three simulated trees at the end of the third year showing stochastic variability and the result of applying simple geometrical hypotheses. 